\title{
Design of the Monitoring System for Subway Platform Screen Doors Based on CAN Bus
}

\author{
Caiqi Hu \\ Department of Mechanical and Electrical Engineering, Qingdao Agricultural University, China, 266109 \\ E-mail: hucaiqi@163.com
}

\begin{abstract}
In this paper, the application layer communication protocol of CAN bus and the monitoring system for subway platform screen doors are proposed. The system framework is built, hardware of every function module for this system is selected, and the monitoring platform of epigynous machine is designed. On the basis of the above work the communication function of the monitoring system is realized, and specific flow chart is given. This system has been tested at a subway line in Shanghai. And the results prove that the system is in full compliance with industry standards and can completely meet the application requirements. The architecture plan of the system is practically proven to be valid and feasible.
\end{abstract}

Keywords: subway platform screen doors, CAN bus, equipments monitoring

Copyright $(2015$ Institute of Advanced Engineering and Science. All rights reserved.

\section{Introduction}

Subway platform screen door is the equipment that located at the subway platform edge, and separated the train operation area from the station public area to protect passengers' safety. The monitoring system of subway platform screen doors refers to that according to the requirements of security levels the various operating information of platform screen doors is real-timely transmitted to the system monitoring center through some form of network bus, and the field equipments are monitored and controlled in real time by the monitoring center. This is one of the important measures for assuring the subway running safely [1-3].

At present the distributed control mode is mostly adopted to the architecture of subway screen doors monitoring system. With the development of technology and the increasing of application requirements some disadvantages of the distributed control system (DCS) emerge. For example, because of no openness of DCS opening, so DCS products of difference manufacturers are not interchangeable, and not interconnection, which limit users' selection of the product. At the same time the decentralized control mode of DCS is not complete, the reliability degree of DCS is not high, and the cost is expensive. Because of the above problems DCS can not well meet customers' demands. With the development of CAN (Controller Area Network) bus technology, which has many advantages, such as multi-master mode, long transmission distance, fast transmission speed, strong anti-interference ability, low cost, good reliability, convenient connection and so on, the design of the monitoring system for subway platform screen doors can have more options [4-7].

In this paper based on CAN bus technology the monitoring system is designed and developed. Advanced technologies in multi fields including controlling, communication, network and circuit design are adopted in this monitoring system. So the system not only has monitoring, alarming, controlling, communication and other functions, but also maximally meets the requirements of high quality, high precision and high reliability. Meanwhile the monitoring system is designed with good scalability and excellent cost-effective.

\section{Architecture Design of the Monitoring System for Platform Screen Doors 2.1. Design of System Hardware}

The monitoring system of field equipments usually consists of three parts: remote monitoring and managing platform of epigynous machine, intelligent node module of 
hypogynous machine, and hardware equipments and communication protocol for network communication between epigynous machine and hypogynous machine.

In this system epigynous machines are mainly composed of industry control computers, which have strong anti-interference ability, and can store information safely and reliably. The mode of $\mathrm{PCl}$ bus and CAN communication card is applied in the communication between epigynous machines and CAN bus. Because the distances from the platform screen doors to the control room are more than $500 \sim 1000 \mathrm{~m}$, and each single side station generally has dozens of screen doors, the quantity of communication data between the screen doors and control computers is very large. ZLG dual PCl-5121 CAN card with high-speed dual port memory is used in the system. This CAN card can improve the data throughput rate, and provide users with library functions, which can conveniently control and operate the card, and bring convenience for the design of software and program in epigynous machine.

The design of controlling hypogynous machine includes the connection between CAN control chip and the microprocessor of MCU or the connection between CAN control chip and PC. The typical intelligent node structure is "MCU\&CAN controller\&CAN driver". In the connection between CAN control chip and PC there is generally a photoelectric isolation circuit. And PHILIPS P8XC591 is used in the CAN controller, which is a microprocessor MCU with CAN controller. PHILIPS PCA82C250 is used in the CAN driver, which can meet the requirement of long distance and low speed data transmission (the longest distance is $10 \mathrm{~km}$, and the lowest speed is $5 \mathrm{kbps}$ ). The system structure diagram is shown in Figure 1.

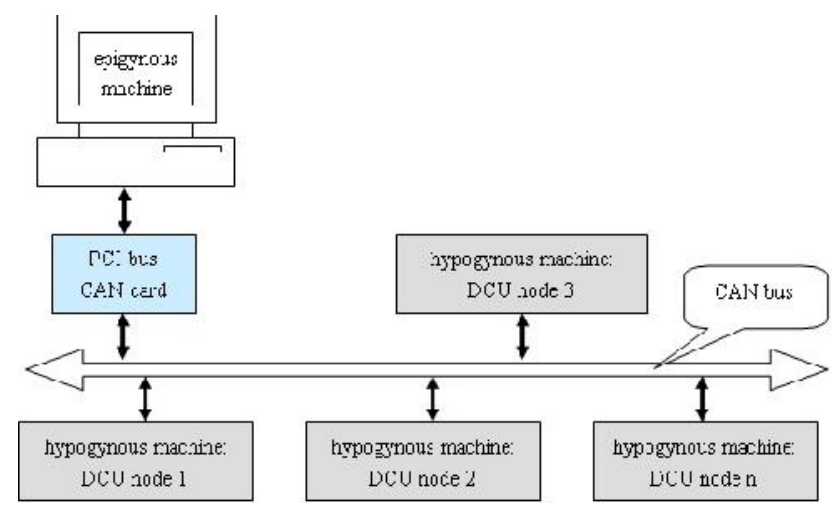

Figure 1. The monitoring system structure diagram of subway platform screen doors

\subsection{Design of System Architecture}

According to the difference of the system function modules, the monitoring system mainly consists of platform screen doors controlling computer (PSC) and signal system (SIG), equipment monitoring and controlling system, automatic fire alarm system (FAS) and interface units of communication clock, and platform end socket control box (PSL), panel system of alarm (PSA), panel of emergency control(PEC), door controlling units (DCU)and the sound and light alarm devices at the top of doors, local control box and so on. In this paper the epigynous machine refers to platform screen doors controlling computer (PSC), and the hypogynous machines are door control units (DCU). The system connection is mainly based on CAN bus connection and hard line connection.

The epigynous machines send commands or data, which are transferred to the network through the CAN communication card, and set parameters and monitor DCU, and real-timely obtain and process the data collected from DCU. The hypogynous machines discriminate themselves identity numbers according to the ID in the commands or data sent to the network, and receive the commands or data corresponding to it. The nodes sending data or commands become bus host station. When multiple DCUs at the same time send commands or data to the host computer, CAN network uses arbitration based on competition, and only permits the node with the highest priority frame turn into the bus host station. So decentralized monitoring and centralized management are integrated into distributed monitoring and control system. The overall architecture diagram of the system is shown in Figure 2. 


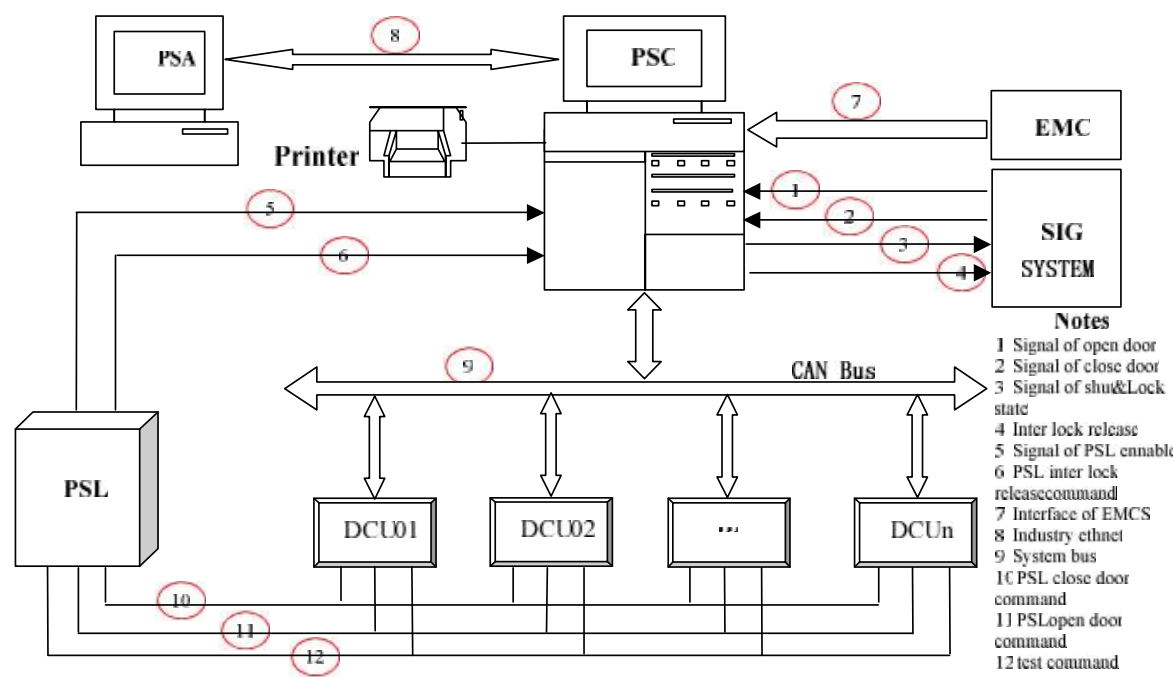

Figure 2. The overall architecture diagram of the system

\section{Design of CAN Bus Communication Protocol 3.1. CAN Bus Communication Protocol}

So far CAN bus is the only field bus which has international standards, and it is a kind of serial communication network that can support distributed control and real-time control. The design of CAN network only uses three layers structure model which conformed to the open system interconnection model $(0 \mathrm{SI})$, the 3 layers are respectively physical layer, data link layer and application layer. The working way of CAN network is multi masters mode. The transmission medium of CAN bus is twisted pair cable, and the fastest communication speed is up to $1 \mathrm{MB} / \mathrm{s}$, the longest direct transmission distance is $10 \mathrm{~km}$. the most important characteristics of CAN protocol is replacing the traditional address coding with communication data block coding. According to the mode of communication data block coding, different nodes can simultaneously receive the same data, and this feature is very useful in the distributed control system. The nodes information on CAN bus is divided into different priorities, which can meet the different real-time requirements. So for CAN bus the data transmission distance is long, and the communication speed is fast, and each frame information is checked by CRC and other measures of error test. Compared with other communication buses the CAN bus has some advantages such as simple structure, stable performance, strong anti-interference ability, good expansibility and openness, and low cost etc.

\subsection{Design of Communication Protocol for the Application Layer}

There are 4 kinds of frame format in CAN protocol communication: data frame, remote frame, error frame and overload frame. Among them the transmission of data frame and remote frame must be under the control of the CPU, and the transmission of error frame and overload frame carries out automatically when errors or overloads occur. When a user want to establish a practical CAN bus communication network, he must draw up the specific application layer protocol. Because in CAN protocol the assignment of information identifiers is not specified, users can use different methods according to different application. It is important to assign CAN identifiers in a communication system based on CAN network. In this system there are many different communications between door control units and epigynous machines, meanwhile each DCU has many different types of information to transfer in the system. According to the different types of information transferred the corresponding levels of the message types are assigned respectively, and the information for recognition also is contained. If reading once data per $100 \mathrm{~ms}$ from every DCU, the transmission speed of the system bus data at the peak time is not more than $10 \mathrm{~K}$ bytes per second. According to the transmission contents and flow of the system, CAN 2.0B protocol is used in the system design. The standard frame and short frame format are used, and the effective quantity of bytes per frame is 8 , in which the transmission time is short. When a node has serious errors, the automatic closing function of the system would cut off the connection between this node and the bus. So other nodes and their 
communications with the bus are not affected, which assures the system with strong antiinterference ability and error detecting ability. The standard frame format of CAN is shown in Table 1.

Table 1. The standard frame format of CAN

\begin{tabular}{|c|c|c|c|c|c|c|c|c|c|}
\hline \multirow[t]{2}{*}{$\begin{array}{l}\text { frame } \\
\text { start }\end{array}$} & \multicolumn{3}{|c|}{ Arbitration field } & \multicolumn{2}{|c|}{ control field } & \multirow{2}{*}{$\begin{array}{c}\text { data field } \\
\text { DataField }\end{array}$} & \multirow[t]{2}{*}{$\begin{array}{l}\text { CRC } \\
\text { field }\end{array}$} & \multirow[t]{2}{*}{$\begin{array}{l}\text { ACK } \\
\text { field }\end{array}$} & \multirow[t]{2}{*}{$\begin{array}{c}\text { frame } \\
\text { end }\end{array}$} \\
\hline & Identifier & RTR & IDE & R0 & DLC & & & & \\
\hline & 4 & & & & 4 & $\hat{\epsilon}$ & & & \\
\hline & 11bits & & & & 4bits & 0 8byte & & & \\
\hline
\end{tabular}

Because the CAN protocol itself has error detection and calibration functions, in the process of the protocol made in this paper, the error detection mechanism is not considered. A byte space in the DataField portion of each transmission frame is reserved for error detection purpose if in the future bus communication fault rate is too high to meet the system requirements. In other words, according to the CAN protocol, the DataField of every transmission frame can transfer no more than 8 bytes data. In this protocol the DataField of every transmission frame uses no more than 7 bytes data and reserved at least 1 bytes space for extending of errors detection.

In the application layer protocol drawn up in this paper, some rules for Identifier of arbitration field and data field of the standard frame format are made and the principle based on nodes is applied to the assignment of identifiers. The format description is shown in Table 2. The low 6 bytes of Identifier are used as identity identification, among them OX00 is identified as the epigynous machine; others are used as the identifiers of DCUs, So one single side station of the system can identify 63 screen doors. The high 5 bytes of Identifier are used as information type numbers, so one screen door can respond to 32 different types of information. DataField is set different byte length along with different information type.

Table 2. Formulations of Identifier and DataField in the standard frame of CAN protocol

\begin{tabular}{|c|c|c|}
\hline Identifier $0-10$ bit & & $\begin{array}{l}\text { DataField } \\
0-7 \text { byte }\end{array}$ \\
\hline number of information type: 5 bits & $\begin{array}{c}\text { identity identifier: } \\
6 \text { bits }\end{array}$ & Information or data \\
\hline
\end{tabular}

\section{Design of System Software}

\subsection{Software Design for Hypogynous Machine}

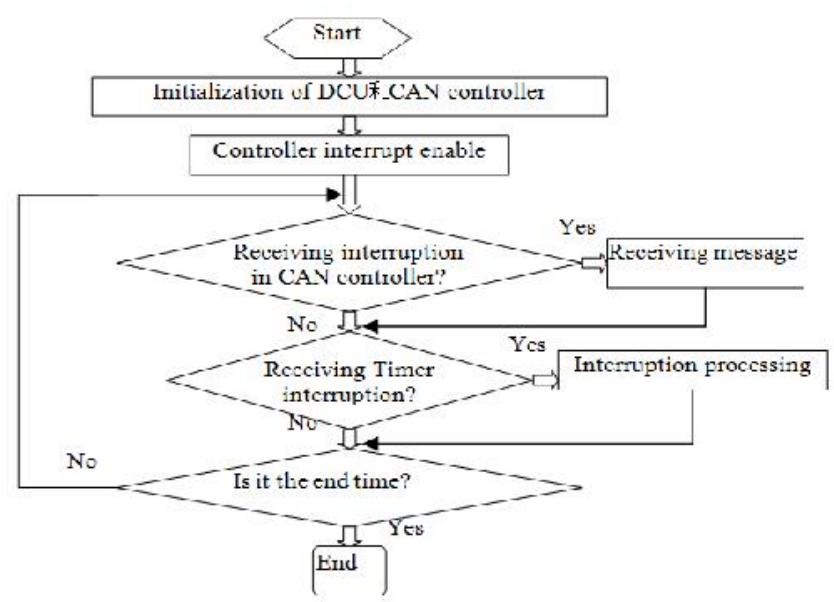

Figure 3. The specific communication procedure of software for hypogynous machine

TELKOMNIKA Vol. 15, No. 3, September 2015: $509-514$ 
CAN controller real-timely monitors the data frame of CAN bus by receiving interrupt signal, and sends the data to node controller, in which the data is mainly correlative information that the epigynous machine sends to hypogynous machine. The specific communication procedure is shown in Figure 3.

\subsection{Software Design for Epigynous Machine}

In this research Windows is used as the system operation platform of monitoring and manage software in the epigynous machine, and Delphi is used as program language for the system software development. The system is developed with function modules including the module of system initialization, the module of massage collecting and processing, the module of remote controlling, the module of displaying and printing trend chart module, the module of inquiring history record, the displaying and printing module of diagram, trend curve, and report and so on.

The epigynous machine monitors CAN bus system, which in nature belongs to multi master field bus. But in this process the epigynous machine node is looked as master control node, which is the combination of master slave mode and multi masters mode. The hypogynous machine sends information only at the time when master control node sends request. The program flow is shown in Figure 4.

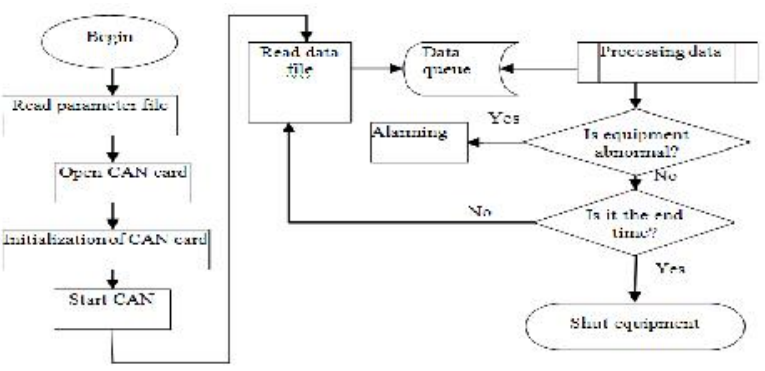

Figure 4. The software flow for epigynous machine

During the design of the software, dynamic interlinking library and multi-thread technologies are applied to read and process the data information of hypogynous machines respectively. The thread of reading data and the thread of processing data share the data information. After being set up and activated the reading data thread continually checks and judges if there is data coming from CAN card or not, if there is the reading data thread would receive the data and put it in the receiving queue. After being set up and activated the processing data thread checks and judges if there is data packet waiting for processing, if there is the processing data thread would read and process the data packet. If there is not data packet in the receiving queue, the processing thread is obstructed until there is data packet coming in the receiving queue. The master monitoring interface of the system is shown in Figure 5 as the following.

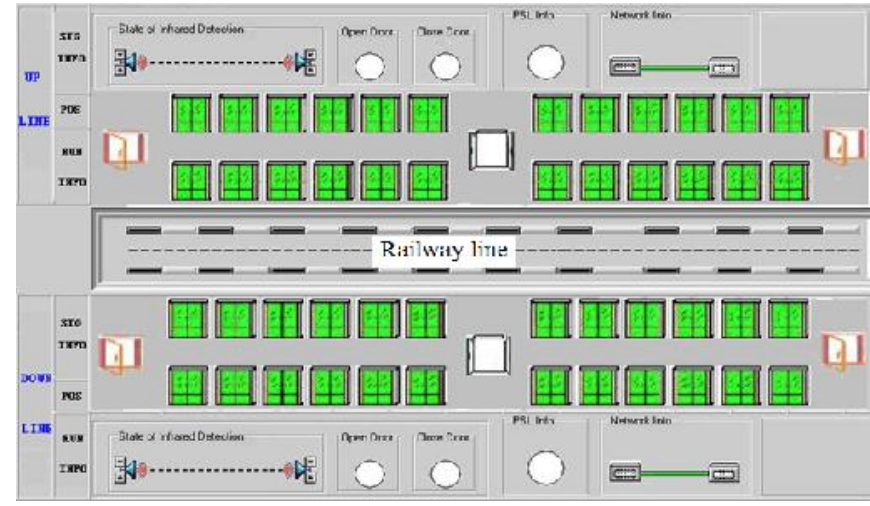

Figure 5. The master monitoring interface of the system 


\subsection{Test Verification}

The monitoring system of subway platform screen doors was tested and ran at Shanghai subway for 3 months and the run fault rate was 0 . The result proves that the monitoring system consisted of software and hardware can entirely meet the requirements of industry standards, and play an important role in protecting passengers' safety. In practice the architecture of the monitoring system is proven to be feasible.

\section{Conclusion}

At present CAN bus technology becomes one of the most popular field bus technologies because it has some advantages such as fast communication speed, good costeffective and high degree of flexibility in network establishment and so on. In this paper the monitoring system of subway platform screen doors is researched and developed based on CAN bus technology, and integrated with the function modules including monitoring, alarming, controlling, displaying, communication and so on. In practice it is proven that the system has good stability, strong anti-interference ability and good openness, as well as the software and hardware of the system designed has good versatility. The function expansion of the system can be conveniently realized along with different application.

\section{References}

[1] Silei Hou, Xiangdong Gong. Design of greenhouse monitoring system gateway based on ZigBee. Microcontroller and Embedded System Application. 2011; (5): 74-76.

[2] Huikuan Yang. Field bus technology and its application. Beijing: Tsinghua University press.1999.

[3] Yuntao Rao, Jijun Zou, Yongyun Zheng. Principle and application technology of CAN field bus. Beijing: Beijing University of Aeronautics and Astronautics Press. 2003.

[4] Dan Wan. Research and application of CAN bus distributed system communication. Wuhan: Huazhong University of Science and Technology. 2009.

[5] Yinhan Li. CAN bus and CAN bus protocol. Field bus and network technology. 2005; 11: 20-23.

[6] Yongbin He, Jianbin Zhou, Dahua Zhang. Design and analysis for the interface control of signal system and screen door system. Study of city rail traffic. 2010; (2): 46-49.

[7] Xiangtian Zhang, Yixiang Wang. Remote Oil valve control system based on GPRS and ZigBee. Microcontroller and embedded system application. 2012; 12(1): 56-58. 